\title{
Structural Characterization of Optimal Event-based Controllers for Linear Stochastic Systems
}

\author{
Adam Molin and Sandra Hirche
}

\begin{abstract}
Recent results in networked control systems indicate substantial benefits of event-based control compared to conventional designs. This paper identifies structural properties of optimal event-based controllers designed for stochastic linear systems. The controller is updated by measurements that are sent over a resource-constrained communication channel. The timings for sending updates are determined by an event-trigger whose decisions are based on noisy measurements. The objective is to design event-triggering mechanisms and controllers that optimally meet a trade-off between control performance and average number of update transmissions. It is shown that the optimal controller is a certainty equivalence controller with an affine linear estimator. The optimal event-trigger consists of a Kalman state estimator and a copy of the state predictor at the controller. The difference between both estimates determines, whether to trigger an update transmission. Numerical simulations illustrate the obtained results.
\end{abstract}

\section{INTRODUCTION}

Recently, there is an increasing interest in designing control systems, which take into account constraints on the information exchange between devices, such as sensors, controllers, and actuators. The development of such networked controllers is urged by the emergence of more and more complex and highly distributed systems. While digital controllers commonly have time-triggered information acquisition schemes, several examples have shown that eventtriggered exchange of information outperforms its timetriggered counterpart [1]-[5].

Contributions can be found in the field of control over communications [1], [2], multi-agent systems [3], [4] and distributed optimization algorithms [5]. All these problems have in common that they must deal with limited exchange of information between engineering entities. In [1], it is shown that an event-triggered impulsive controller is capable of reducing the state variance significantly compared to a timetriggered minimum variance controller, while both having the same average transmission rate. The work in [2] considers multiple independent control loops which share a common digital network. There, it turns out that an event-triggered scheduling scheme outperforms a time-triggered scheduling scheme in terms of aggregate state variance. In the field of multi-agent systems, it is shown in [3] that an event-triggered control strategy reduces the need of communication significantly while maintaining a certain degree of connectivity.

Whereas the objective of these motivating examples is to show the benefits of event-triggered control schemes, there

A. Molin and S. Hirche are with the Institute of Automatic Control Engineering, Technische Universität München, Arcisstraße 21 D-80290 München, Germany; http://www.lsr.ei.tum.de, adam.molinetum. de, hirchedtum. de is no concrete specification of how to choose optimally the rules when an event occurs. The focus of this paper is to design event-triggered controllers for a networked control system, where sensor measurements are sent over a network to the controller. An event-triggered controller consists of a (i) control policy that applies control inputs from available observations and (ii) a scheduling policy assigning transmission timings. The goal is to minimize an objective function consisting of a standard quadratic control cost and a communication cost penalizing information exchange between sensor and controller. The choice for this cost function is inspired by related work for estimation problems with limited communication capabilities [6], [7]. In [8], [9], optimal event-triggered controllers are obtained, when limiting the number of transmissions for a finite interval and restricting the controller to be time-invariant. Other related results appear in [10], [11], which consider discrete-time systems with a hard constraint on the number of transmissions. In contrast to that, this paper penalizes the average number of transmissions which is reflected within the cost function.

The main contribution of this paper is to obtain structural properties of the optimal event-triggered controller that minimizes the underlying cost function. The obtained results extend the results of [12] to the case of noisy observations at the event-trigger. It is shown that the optimal event-triggered controller consists of an affine linear controller, where the gains can be obtained by standard methods. The optimal event-triggering law is a policy depending on the discrepancy of state estimate at the controller and at the event-trigger. It turns out that transmitting the Kalman state estimate instead of the complete observation history at the event-trigger still yields the optimal cost.

Although these structural properties seem to be intuitive, a rigorous derivation of these poses the need of innovative reformulation techniques. This is mainly due to the different information available at controller and event-trigger. The reformulation techniques constitute the key tool for having new insights into the proposed problem and for enabling the use of standard optimization methods. It should be mentioned that this kind of the optimal event-triggered controller have already been proposed in other works. There, they resulted from heuristics [13] or restricting assumptions [6], [14]. Therefore, the results derived in this paper support these assumptions by giving an interpretation in terms of optimality.

The remainder of the paper is organized into four sections. Section II introduces the system model and gives the problem statement. In section III, the underlying problem is reformulated and a structural characterization of the optimal 
solution is given for the controller and the event-trigger, respectively. Finally, a numerical example illustrates the solution algorithm for finding the optimal event-triggered controller in section IV.

Notation. In this paper, the operators $\operatorname{tr}[\cdot]$ and $(\cdot)^{\mathrm{T}}$ denote the trace and the transpose operator of a square matrix, respectively. The variable $P$ denotes the probability measure on the abstract sample space denoted by $\Omega$. The expectation operator is denoted by $E[\cdot]$ and the conditional expectation is denoted by $\mathrm{E}[\cdot \mid \cdot]$.

\section{PROBLEM STATEMENT}

We consider the following stochastic time-invariant discrete-time system $\mathcal{P}$

$$
\begin{aligned}
x_{k+1} & =A x_{k}+B u_{k}+w_{k}, \\
y_{k} & =C x_{k}+v_{k},
\end{aligned}
$$

where $A \in \mathbb{R}^{n \times n}, B \in \mathbb{R}^{n \times d}$ and $C \in \mathbb{R}^{m \times n}$. The variables, $x_{k}, u_{k}$ and $y_{k}$ denote the state, the control input and measurement and are taking values in $\mathbb{R}^{n}, \mathbb{R}^{d}$ and $\mathbb{R}^{m}$, respectively. The system noise $w_{k}$ and measurement noise $v_{k}$ take values in $\mathbb{R}^{n}$ and $\mathbb{R}^{m}$, respectively, and are i.i.d. (independent identically distributed) zero-mean Gaussian distributed sequences with identity covariance matrix. The initial state, $x_{0}$ is Gaussian with mean $x_{0}^{\mathrm{m}}$ and covariance $S=\mathrm{E}\left[\left(x_{0}-x_{0}^{\mathrm{m}}\right)\left(x_{0}-x_{0}^{\mathrm{m}}\right)^{\mathrm{T}}\right]$.

Remark 1: It is straight forward to extend all results to time-invariant systems and arbitrary noise covariance matrices. The chosen restriction facilitates the illustration of results without loosing generality.

We assume that system parameters and statistics are known to the event-trigger and the controller. The event-trigger $\mathcal{E}$ situated at the sensor side has access to the complete observation history and decides, whether the controller $\mathcal{C}$ should receive an update over the network denoted by $\mathcal{N}$. The controller calculates inputs $u_{k}$ to regulate process $\mathcal{P}$. The system model is illustrated in Figure 1.

The event-trigger output $\delta_{k} \in\{0,1\}$ is defined as follows:

$$
\delta_{k}= \begin{cases}1 & \text { update is sent } \\ 0 & \text { otherwise }\end{cases}
$$

If the event-trigger decides to update the controller, all past measurements starting from the last update transmission are sent to the controller. Transmission of all past measurements between two update timings constitutes a preliminary assumption which is relaxed in section III-B. Hence,

$$
z_{k}= \begin{cases}\left(y_{\tau_{k}+1}, \ldots, y_{k}\right), & \delta_{k}=1 \\ \emptyset, & \delta_{k}=0\end{cases}
$$

with

$$
\tau_{k}=\max \left\{l \mid \delta_{l}=1, l<k\right\}
$$

being the last time step, where an update has been transmitted. In case no transmission has occurred, we define $\tau_{k}=-1$.

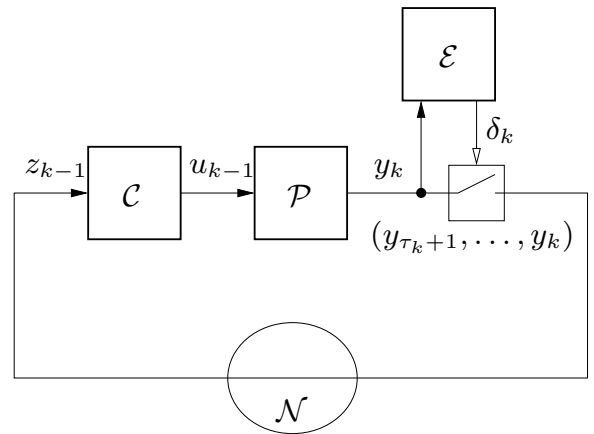

Fig. 1. System model of the networked control system with plant $\mathcal{P}$, event-trigger $\mathcal{E}$, controller $\mathcal{C}$ and communication network $\mathcal{N}$.

Remark 2: It should be noted that sending no update is still valuable information for the controller. This will play a crucial role in section III-B.

The design objective is to find admissible event-triggering policies $\mathrm{f}$ and control policies $\gamma$ that minimize the finitehorizon criterion

$$
J(\mathrm{f}, \gamma)=\mathrm{E}\left[x_{N}^{\mathrm{T}} Q_{N} x_{N}+\sum_{k=0}^{N-1} x_{k}^{\mathrm{T}} Q x_{k}+u_{k}^{\mathrm{T}} R u_{k}+\lambda \delta_{k}\right]_{(3)}
$$

The weighting matrices $Q, Q_{N}$ are positive definite and $R$ is positive semi-definite. The positive factor $\lambda$ can be regarded as the weight of penalizing information exchange between sensor and controller. The admissible policies for the eventtrigger and the controller at time $k$ are defined as Borelmeasurable functions of their past available data

$$
\begin{aligned}
\delta_{k} & =\mathrm{f}_{k}\left(\mathcal{I}_{k}^{\mathcal{E}}\right), \\
u_{k} & =\gamma_{k}\left(\mathcal{I}_{k}^{\mathcal{C}}\right) .
\end{aligned}
$$

The information patterns $\mathcal{I}_{k}^{\mathcal{E}}$ and $\mathcal{I}_{k}^{\mathcal{C}}$ of the event-trigger and controller are defined as

$$
\begin{aligned}
& \mathcal{I}_{k}^{\mathcal{E}}=\left\{y_{0}, \delta_{0}, u_{0}, y_{1}, \ldots, y_{k-1}, \delta_{k-1}, u_{k-1}, y_{k}\right\}, \\
& \mathcal{I}_{k}^{\mathcal{C}}=\left\{\delta_{0}, z_{0}, u_{0}, \ldots, \delta_{k-1}, z_{k-1}, u_{k-1}, \delta_{k}, z_{k}\right\} .
\end{aligned}
$$

With a slight abuse of notation, we use $\mathcal{I}_{k}^{\mathcal{E}}$ and $\mathcal{I}_{k}^{\mathcal{C}}$ as sets, when referring to information patterns, and as vectors, when referring to the information state. Apart from this, we will often consider the history of a specific signal up to time $k$. These are summarized into vectors denoted by

$$
\begin{aligned}
U^{k}=\left[u_{0}^{\mathrm{T}}, \ldots, u_{k}^{\mathrm{T}}\right]^{\mathrm{T}}, & Y^{k}=\left[y_{0}^{\mathrm{T}}, \ldots, y_{k}^{\mathrm{T}}\right]^{\mathrm{T}}, \\
W^{k}=\left[w_{0}^{\mathrm{T}}, \ldots, w_{k}^{\mathrm{T}}\right]^{\mathrm{T}}, & V^{k}=\left[v_{0}^{\mathrm{T}}, \ldots, v_{k}^{\mathrm{T}}\right]^{\mathrm{T}} .
\end{aligned}
$$

It can be seen from the definition of $\mathcal{I}_{k}^{\mathcal{E}}$ that control inputs are assumed to be known to the event-trigger. In the following, we assume our system has a side information channel communicating the control inputs to the event-trigger as indicated in Figure 2. It will be observed in section III-C that control inputs can indeed be calculated at the eventtriggering unit without the additional side channel. 


\section{STRUCTURAL CHARACTERIZATION}

This section is divided into three subsections. First, we investigate the form of the optimal controller. This urges us to characterize the least-squares state estimators at the eventtrigger and controller. Finally, the obtained results allow us to specify the form of optimal event-triggers.

\section{A. Structure of the optimal controller}

In order to derive a structural characterization of the optimal control policy, we first consider a related, but slightly different problem, where the event-trigger is prespecified and the optimal control law is to be found. The resulting solution will yield important insights to the original problem.

The prespecified event-trigger is constructed by admissible policy $\overline{\mathrm{f}}$ and a transformation $\mathcal{T}_{\bar{\gamma}}$ which is parameterized by an admissible control policy $\bar{\gamma}$. The applied $\delta_{k}$ is fed into $\mathcal{T}_{\bar{\gamma}}$ through a one-step delay element denoted by $\mathrm{T}$. The system with prespecified event-trigger is called reformulated system in the following and is illustrated in Figure 2. The aim is to find the optimal control policy $\gamma^{*}$ for this system minimizing the cost function given by (3).

The transformation $\mathcal{T}_{\bar{\gamma}}$ recalculates measurement output and control input that would result, if $\bar{\gamma}$ and $\overline{\mathrm{f}}$ were used in the original problem. These variables are denoted by $\bar{y}_{k}$ and $\bar{u}_{k}$. It can be shown that $\mathcal{T}_{\bar{\gamma}}$ is given by the following equations:

$$
\begin{aligned}
& \bar{y}_{k}=y_{k}+\sum_{m=0}^{k-1} C A^{k-m-1} B\left(\bar{\gamma}_{m}\left(\bar{z}_{0}, \ldots, \bar{z}_{m}\right)-u_{m}\right), \\
& \bar{z}_{k}= \begin{cases}\left(\bar{y}_{\tau_{k}+1}, \ldots, \bar{y}_{k}\right), & \delta_{k}=1, \\
\emptyset, & \delta_{k}=0,\end{cases} \\
& \bar{u}_{k}=\bar{\gamma}_{k}\left(\bar{z}_{0}, \ldots, \bar{z}_{k}\right) .
\end{aligned}
$$

In other words, transformation $\mathcal{T}_{\bar{\gamma}}$ emulates the closed-loop behavior of a system with policies $\bar{\gamma}$ and $\overline{\mathrm{f}}$.

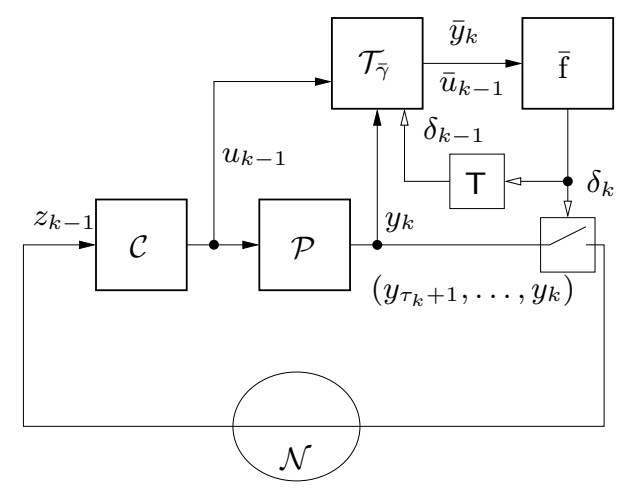

Fig. 2. Reformulated system model with transformation $\mathcal{T}_{\bar{\gamma}}$, one-step delay element $\mathrm{T}$ and prespecified policies $\bar{\gamma}$ and $\overline{\mathrm{f}}$.

The event-triggering law $\overline{\mathrm{f}}_{k}$ decides upon past data $\bar{Y}^{k}, \bar{U}^{k-1}$ and $\delta^{k-1}$, whether or not to transmit an update at time $k$. An obvious observation is that choosing $\bar{\gamma}$ as the control law in the reformulated system yields exactly the original system with policies $\bar{\gamma}$ and $\bar{f}$. The key property within the reformulated system is that for each sample path $\omega \in \Omega$, the sequence $\left(\delta_{k}\right)_{k}$ will be the same independent of the control inputs chosen. By definition of transformation $\mathcal{T}_{\bar{\gamma}}$ in (5), we observe that

$$
\begin{aligned}
\bar{y}_{0} & =y_{0}=C\left(x_{0}+w_{-1}\right)+v_{0} \\
\bar{y}_{1} & =C\left(A x_{0}+w_{0}\right)+v_{1}+C B \bar{\gamma}\left(\bar{z}_{0}\right) \\
& \vdots \\
\bar{y}_{k} & =C\left(A^{k} x_{0}+\sum_{m=0}^{k-1} A^{k-m-1}\left(B \bar{\gamma}_{m}\left(\bar{z}_{0}, \ldots, \bar{z}_{m}\right)+w_{m}\right)\right)+v_{k} .
\end{aligned}
$$

This implies that $\bar{y}_{k}, \bar{z}_{k}$ and $\bar{u}_{k}$ can be expressed by primitive random variables, which shows that $\delta_{k}$ is a function of primitive variables and independent of the control inputs $u_{k}$. This key feature is used for showing the following lemma.

Lemma 1: The optimal control policy $\gamma^{*}$ minimizing cost function $J$ from (3) for the reformulated system is given by

$$
u_{k}=\gamma_{k}^{*}\left(\mathcal{I}_{k}^{\mathcal{C}}\right)=-L_{k} \mathrm{E}\left[x_{k} \mid \mathcal{I}_{k}^{\mathcal{C}}\right]
$$

with

$$
\begin{aligned}
L_{k}= & \left(R+B^{\mathrm{T}} P_{k+1} B\right)^{-1} B^{\mathrm{T}} P_{k+1} A, \\
P_{k}= & A^{\mathrm{T}} P_{k+1} A+Q-A^{\mathrm{T}} P_{k+1} B \\
& \times\left(R+B^{\mathrm{T}} P_{k+1} B\right)^{-1} B^{\mathrm{T}} P_{k+1} A, \\
P_{N}= & Q_{N},
\end{aligned}
$$

where $P_{k} \in \mathbb{R}^{n \times n}$ is non-negative definite for all $k$.

Proof: Due to the fact that the sequence $\left(\delta_{k}\right)_{k}$ is independent of the sequence of control inputs for each sample path $\omega \in \Omega$, the term $E\left[\sum_{k=0}^{N-1} \lambda \delta_{k}\right]$ in Equation (3) is constant and can be omitted from the optimization. Similarly to [15], we show that there is a function $\Delta_{k}=x_{k}-\mathrm{E}\left[x_{k} \mid \mathcal{I}_{k}^{\mathcal{C}}\right]$ of the primitive random variables which does not depend on the policy $\gamma$ being used. The internal states of the eventtrigger may be disregarded as (i) they do not contribute to the cost function and (ii) the control input does not influence the evolution of output $\delta_{k}$. We fix a policy $\gamma$ and consider two types of the reformulated system: The forced system model is given by (1), whereas the unforced system model with zero inputs reads

$$
\tilde{x}_{k+1}=A \tilde{x}_{k}+\tilde{w}_{k}, \quad \tilde{y}_{k}=C \tilde{x}_{k}+\tilde{v}_{k} .
$$

We assume both have the same evolution of primitive random variable, i.e.

$$
x_{0}=\tilde{x}_{0}, \quad w_{k}=\tilde{w}_{k}, \quad v_{k}=\tilde{v}_{k}, \quad k=0, \ldots, N-1 .
$$

The received signal at the controller is given by (2) for the forced system and

$$
\tilde{z}_{k}= \begin{cases}\left(\tilde{y}_{\tilde{\tau}_{k}+1}, \ldots, \tilde{y}_{k}\right), & \tilde{\delta}_{k}=1 \\ \emptyset, & \tilde{\delta}_{k}=0\end{cases}
$$

for the unforced system. Due to linearity, we can rewrite the forced and the unforced to the following form

$$
\begin{array}{r}
x_{k}=F_{k} x_{0}+G_{k} U^{k-1}+H_{k} W^{k-1} \\
\tilde{x}_{k}=F_{k} x_{0}+H_{k} W^{k-1}
\end{array}
$$


where $U^{k-1} W^{k-1}$ are the augmented vectors defined in (4) and $F_{k}, G_{k}$ and $H_{k}$ are appropriate matrices constructed from $A, B$ and $k$. As $U^{k-1}$ is measurable with respect to the information pattern $\mathcal{I}_{k}^{\mathcal{C}}$, the conditional expectations are

$$
\begin{aligned}
& \mathrm{E}\left[x_{k} \mid \mathcal{I}_{k}^{\mathcal{C}}\right]=F_{k} \mathrm{E}\left[x_{0} \mid \mathcal{I}_{k}^{\mathcal{C}}\right]+G_{k} U^{k-1}+H_{k} \mathrm{E}\left[W^{k-1} \mid \mathcal{I}_{k}^{\mathcal{C}}\right] \\
& \mathrm{E}\left[\tilde{x}_{k} \mid \mathcal{I}_{k}^{\mathcal{C}}\right]=F_{k} \mathrm{E}\left[x_{0} \mid \mathcal{I}_{k}^{\mathcal{C}}\right]+H_{k} \mathrm{E}\left[W^{k-1} \mid \mathcal{I}_{k}^{\mathcal{C}}\right]
\end{aligned}
$$

The output vector $\tilde{Y}^{k}$ of the unforced system can be expressed by

$$
\tilde{Y}^{k}=Y^{k}-R_{k} U^{k-1}=C F_{k} x_{0}+S_{k} W^{k-1}+T_{k} V^{k-1}
$$

where $R_{k}, S_{k}$ and $T_{k}$ are appropriate matrices. As the reformulated system has a fixed sequence $\left(\delta^{k}\right)_{k}$ independent of the control inputs chosen, we state

$$
\tilde{\delta}_{k}=\delta_{k} \quad \text { and } \quad \tilde{\tau}_{k}=\tau_{k}, \quad k=0, \ldots, N-1 .
$$

Hence,

$\tilde{Z}^{k}=\left[\tilde{Y}^{\tau_{k+1}}, \emptyset, \ldots, \emptyset\right]=\left[Y^{\tau_{k+1}}-R_{\tau_{k+1}} U^{\tau_{k+1}-1}, \emptyset, \ldots, \emptyset\right]$.

By (8) and (9), we observe that $\tilde{Z}^{k}$ only depends on the primitive variables. Equation (10) implies that $\mathrm{E}\left[\tilde{x}_{k} \mid \mathcal{I}_{k}^{\mathcal{C}}\right]=\mathrm{E}\left[\tilde{x}_{k} \mid \tilde{Z}^{k}, \tilde{\delta}^{k}\right]$ and we have

$$
\Delta_{k}=\tilde{x}_{k}-\mathrm{E}\left[\tilde{x}_{k} \mid \tilde{Z}^{k}, \tilde{\delta}^{k}\right] .
$$

Accordingly, the function $\Delta_{k}=\tilde{x}_{k}-E\left[\tilde{x}_{k} \mid \tilde{Z}^{k}, \tilde{\delta}^{k}\right]$ is a function of the primitive random variables and is independent on the control policy used.

Having this result and using the fact that the objective function is a standard quadratic cost term, we can proceed along the same lines as in section 5.2 in [15] to obtain the optimal controller stated in above lemma.

Remark 3: The gains $L_{k}$ correspond to the optimal control gains of the standard LQG problem.

The result in Lemma 1 for the reformulated system can be used to characterize the optimal control law of the original problem. This is summarized in Lemma 2.

Lemma 2: The optimal control law $\gamma^{*}$ minimizing cost function $J$ given by (3) for the original system has the same form as the optimal control law for the reformulated system.

Proof: The reformulated problem with prespecified event-trigger constructed by $\bar{\gamma}$ and $\bar{f}$ considers a subset of admissible policy pairs $(\gamma, f)$ which is parameterized by admissible control laws $\gamma$. In fact, this subset can be viewed as an equivalence class defined by the following equivalence relation. Two policies, $\left(\gamma^{\mathrm{A}}, \mathrm{f}^{\mathrm{A}}\right)$ and $\left(\gamma^{\mathrm{B}}, \mathrm{f}^{\mathrm{B}}\right)$ are equivalent if and only if

$$
\left(\delta_{k}^{\mathrm{A}}\right)_{k}=\left(\delta_{k}^{\mathrm{B}}\right)_{k}, \quad \forall \omega \in \Omega .
$$

Having fixed a control policy $\gamma$ in the reformulated problem, the system resembles the original system with policy pair $\left(\gamma, \overline{\mathrm{f}} \circ \mathcal{T}_{\bar{\gamma}}\right)$ and therefore, both have the same costs. The composition operator $\circ$ is applied for every time step $k$. A basic property of equivalence classes is that the whole solution space is partitioned by the equivalence relation. As the structure of the optimal controller structure given by Lemma 1 is invariant to the specific equivalence class chosen, the optimal law $\gamma^{*}$ given by (6) is optimal for our original problem within the complete solution space. This concludes the proof.

\section{B. Structure of the optimal state estimator}

It has been shown in the last section that the optimal controller is a certainty equivalence controller consisting of linear gains $L_{k}$ that can be computed in advance and a state estimator $\mathrm{E}\left[x_{k} \mid \mathcal{I}_{k}^{\mathcal{C}}\right]$. The aim of this paragraph is to specify the form of $\mathrm{E}\left[x_{k} \mid \mathcal{I}_{k}^{\mathcal{C}}\right]$.

For notational convenience, we define

$$
\begin{aligned}
\hat{x}_{k \mid k}^{\mathcal{E}} & =\mathrm{E}\left[x_{k} \mid \mathcal{I}_{k}^{\mathcal{E}}\right], \\
\hat{x}_{k \mid k-1}^{\mathcal{E}} & =\mathrm{E}\left[x_{k} \mid \mathcal{I}_{k-1}^{\mathcal{E}}\right], \\
\hat{x}_{k \mid k}^{\mathcal{C}} & =\mathrm{E}\left[x_{k} \mid \mathcal{I}_{k}^{\mathcal{C}}\right]
\end{aligned}
$$

with $\hat{x}_{0 \mid-1}^{\mathcal{E}}=\mathrm{E}\left[x_{0}\right]$ and the error covariance matrices

$$
\begin{aligned}
\Sigma_{k \mid k}^{\mathcal{E}} & =\mathrm{E}\left[\left(x_{k}-\hat{x}_{k \mid k}^{\mathcal{E}}\right)\left(x_{k}-\hat{x}_{k \mid k}^{\mathcal{E}}\right)^{\mathrm{T}}\right], \\
\Sigma_{k \mid k-1}^{\mathcal{E}} & =\mathrm{E}\left[\left(x_{k}-\hat{x}_{k \mid k-1}^{\mathcal{E}}\right)\left(x_{k}-\hat{x}_{k \mid k-1}^{\mathcal{E}}\right)^{\mathrm{T}}\right] .
\end{aligned}
$$

Lemma 3: The optimal state estimator at the event-trigger is given by the Kalman filter

$$
\begin{aligned}
\hat{x}_{k \mid k}^{\mathcal{E}}= & A \hat{x}_{k-1 \mid k-1}^{\mathcal{E}}+B u_{k-1} \\
& +\Sigma_{k \mid k}^{\mathcal{E}} C^{\mathrm{T}}\left(y_{k}-C A \hat{x}_{k-1 \mid k-1}^{\mathcal{E}}-C B u_{k-1}\right)
\end{aligned}
$$

independently of the scheduling law chosen.

Proof: Fix a scheduling law f. As the variable $\delta_{k}$ is a function of $U^{k-1}$ and $Y^{k}$, it does not contain additional information. Hence, we have

$$
\hat{x}_{k \mid k}^{\mathcal{E}}=\mathrm{E}\left[x_{k} \mid U^{k-1}, Y^{k}\right] .
$$

Given the control inputs $U^{k-1}$, the state $x_{k}$ and past observations $Y^{k}$ are jointly Gaussian. This is due to the fact that the primitive random variable are Gaussian and $x_{k}$ and $y_{k}$ are linear functions of them. Therefore, the least-squares estimator $\mathrm{E}\left[x_{k} \mid U^{k-1}, Y^{k}\right]$ is linear and can be computed by the Kalman filter given by (11).

Remark 4: The covariance matrix $\Sigma_{k \mid k}^{\mathcal{E}}$ appearing in (11) is computed in advance by

$$
\begin{aligned}
& \Sigma_{k \mid k}^{\mathcal{E}}=\Sigma_{k \mid k-1}^{\mathcal{E}}-\Sigma_{k \mid k-1}^{\mathcal{E}} C^{\mathrm{T}}\left(C \Sigma_{k \mid k-1}^{\mathcal{E}} C^{\mathrm{T}}+I\right)^{-1} C \Sigma_{k \mid k-1}^{\mathcal{E}} \\
& \Sigma_{k+1 \mid k}^{\mathcal{E}}=A \Sigma_{k \mid k}^{\mathcal{E}} A^{\mathrm{T}}+I, \quad \Sigma_{0 \mid-1}^{\mathcal{E}}=S .
\end{aligned}
$$

Determining the optimal state estimator at the controller $\hat{x}_{k \mid k}^{\mathcal{C}}$ turns out to be a more difficult task, as (i) it is dependent on the scheduling law and (ii) non-Gaussian observations are to be incorporated. Despite these difficulties, it is possible to give a characterization of $\hat{x}_{k \mid k}^{\mathcal{C}}$ stated in the following lemma.

Lemma 4: Let the event-triggering law $\mathrm{f}$ be fixed. Then, the optimal state estimator at the controller takes the form

$$
\hat{x}_{k \mid k}^{\mathcal{C}}= \begin{cases}\hat{x}_{k \mid k}^{\mathcal{E}} & \delta_{k}=1 \\ A \hat{x}_{k-1 \mid k-1}^{\mathcal{C}}+B u_{k-1}+\alpha_{\tau_{k}, k} & \delta_{k}=0\end{cases}
$$


where the bias term $\alpha_{\tau_{k}, k} \in \mathbb{R}^{n}$ depends on the eventtriggering law $\mathrm{f}$.

Proof: In case of $\delta_{k}=1$, we have $\mathcal{I}_{k}^{\mathcal{C}}=\mathcal{I}_{k}^{\mathcal{E}}$ because of $Z^{k}=Y^{k}$. This implies that $\hat{x}_{k \mid k}^{\mathcal{C}}=\hat{x}_{k \mid k}^{\mathcal{E}}$ for $\delta_{k}=1$.

In case of $\delta_{k}=0$, we first consider $\tau_{k}=k-1$, i.e. $\hat{x}_{k-1 \mid k-1}^{\mathcal{C}}=\hat{x}_{k-1 \mid k-1}^{\mathcal{E}}$. Due to the tower property of the conditional expectation, we have

$$
\begin{aligned}
\mathrm{E}\left[x_{k} \mid \mathcal{I}_{k}^{\mathcal{C}}, \delta_{k}=0\right] & =\mathrm{E}\left[\hat{x}_{k \mid k}^{\mathcal{E}} \mid \mathcal{I}_{k}^{\mathcal{C}}, \delta_{k}=0\right] \\
& =\mathrm{E}\left[A \hat{x}_{k-1 \mid k-1}^{\mathcal{E}}+B u_{k-1}+\Sigma_{k \mid k}^{\mathcal{E}} C^{\mathrm{T}}\left(y_{k}-\right.\right. \\
& \left.\left.-C A \hat{x}_{k-1 \mid k-1}^{\mathcal{E}}-C B u_{k-1}\right) \mid \mathcal{I}_{k-1}^{\mathcal{E}}, \delta_{k}=0\right] \\
& =A \hat{x}_{k-1 \mid k-1}^{\mathcal{E}}+B u_{k-1}+\Sigma_{k \mid k}^{\mathcal{E}} C^{\mathrm{T}} \mathrm{E}\left[y_{k}-\right. \\
& \left.\left.-C A \hat{x}_{k-1 \mid k-1}^{\mathcal{E}}-C B u_{k-1}\right) \mid \mathcal{I}_{k-1}^{\mathcal{E}}, \delta_{k}=0\right]
\end{aligned}
$$

The conditional expectation can be split into two terms

$$
\begin{aligned}
& \mathrm{E}\left[y_{k}-C\left(A \hat{x}_{k-1 \mid k-1}^{\mathcal{E}}-B u_{k-1}\right) \mid \mathcal{I}_{k-1}^{\mathcal{E}}, \delta_{k}=0\right]= \\
& \quad=C A \mathrm{E}\left[x_{k-1}-\hat{x}_{k-1 \mid k-1}^{\mathcal{E}} \mid \mathcal{I}_{k-1}^{\mathcal{E}}, \delta_{k}=0\right] \\
& \quad+\mathrm{E}\left[C w_{k-1}+v_{k} \mid \delta_{k}=0\right]
\end{aligned}
$$

Applying the tower property again to the first term yields

$$
\begin{aligned}
& \mathrm{E}\left[x_{k-1}-\hat{x}_{k-1 \mid k-1}^{\mathcal{E}} \mid \mathcal{I}_{k-1}^{\mathcal{E}}, \delta_{k}=0\right] \\
& \quad=\mathrm{E}\left[\mathrm{E}\left[x_{k-1} \mid \mathcal{I}_{k}^{\mathcal{E}}\right]-\hat{x}_{k-1 \mid k-1}^{\mathcal{E}} \mid \mathcal{I}_{k-1}^{\mathcal{E}}, \delta_{k}=0\right]
\end{aligned}
$$

Due to Corollary E.3.7 from [15], we know that

$$
\mathrm{E}\left[x_{k-1} \mid \mathcal{I}_{k}^{\mathcal{E}}\right]-\hat{x}_{k-1 \mid k-1}^{\mathcal{E}}=\mathrm{E}\left[x_{k-1} \mid y_{k}-\mathrm{E}\left[y_{k} \mid Y^{k-1}\right]\right]-\mathrm{E}\left[x_{k-1}\right]
$$

Therefore, above term is independent of past observations $\mathcal{I}_{k-1}^{\mathcal{E}}$. By defining

$$
\begin{aligned}
\alpha_{k-1, k}= & \mathrm{E}\left[C A \hat{x}\left(y_{k}-\mathrm{E}\left[y_{k} \mid Y^{k-1}\right]\right)\right. \\
& \left.+C w_{k-1}+v_{k} \mid \delta_{k}=0\right]-E\left[x_{k}\right],
\end{aligned}
$$

we have proved above lemma for $\tau_{k}=k-1$. By fixing the scheduling law $\mathrm{f}$, all variables have a unique probability distribution which enables the computation of $\alpha_{k-1, k}$. It is possible to obtain similar results for $\tau_{k} \in\{-1, \ldots, k-2\}$, but this is omitted due to page limitation.

Remark 5: The bias $\alpha_{\tau_{k}, k}$ can be interpreted as a correction term to enhance the state estimate at the controller, when incorporating additional information $\delta_{\tau_{k}}=\cdots=\delta_{k}=0$.

Remark 6: As a side result of Lemma 4, it can be seen from (12) that it suffices to transmit the state estimate $\hat{x}_{k \mid k}^{\mathcal{E}}$ instead of the complete sequence $\left(y_{\tau_{k}+1}, \ldots, y_{k}\right)$. This is advantageous from a technological point of view, because it roughly states that the amount of information to be transmitted is independent of the time elapsed between two subsequent transmissions.

\section{Structure of the optimal event-trigger}

Based on the results of the preceding paragraphs, we investigate the form of the optimal event-triggering law in this subsection. Therefore, we rewrite the optimization problem taking into account Lemma 1 and 2. By using an identity from [16] the cost function defined in (3) can be written as

$$
\begin{aligned}
& J(\mathrm{f}, \gamma)=\mathrm{E}\left[x_{0}^{\mathrm{T}} P_{0} x_{0}+\sum_{k=0}^{N-1} w_{k}^{\mathrm{T}} P_{k+1} w_{k}\right. \\
& \left.+\sum_{k=0}^{N-1}\left(u_{k}+L_{k} x_{k}\right)^{\mathrm{T}}\left(R+B^{\mathrm{T}} P_{k+1} B\right)\left(u_{k}+L_{k} x_{k}\right)+\lambda \delta_{k}\right],
\end{aligned}
$$

where $L_{k}$ and $P_{k}$ are given by Lemma 1 .

Taking into account the optimal control law $\gamma^{*}$ given by (6) and Lemma 2, we obtain the following optimization problem

$$
\min _{f} \mathrm{E}\left[\sum_{k=0}^{N-1}\left(x_{k}-\hat{x}_{k \mid k}^{\mathcal{C}}\right)^{\mathrm{T}} \Gamma_{k}\left(x_{k}-\hat{x}_{k \mid k}^{\mathcal{C}}\right)+\lambda \delta_{k}\right],
$$

where

$$
\Gamma_{k}=L_{k}^{\mathrm{T}}\left(R+B^{\mathrm{T}} P_{k+1} B\right) L_{k}, \quad k=0, N-1 .
$$

As the term $\mathrm{E}\left[x_{0}^{\mathrm{T}} P_{0} x_{0}+\sum_{k=0}^{N-1} w_{k}^{\mathrm{T}} P_{k+1} w_{k}\right]$ is constant, it can be omitted from the optimization.

The fact that $\hat{x}_{k \mid k}^{\mathcal{C}}$ is dependent on the event-triggering law $f$ restrains us from applying the dynamic programming algorithm to problem (13). Lemma 4 states that this dependence appears within the parameters $\alpha_{\tau_{k}, k}$.

When fixing an event-triggering policy $\mathrm{f}$, we are able to calculate bias terms $\alpha_{\tau_{k}, k}$ by Lemma 4 . Subsequently, we proceed the other way round by fixing bias terms $\alpha_{\tau_{k}, k}$ and calculating the optimal $\mathrm{f}$ for such configuration. When minimizing over all possible configurations of $\alpha_{\tau_{k}, k}$, we also obtain the optimal event-triggering law $f$. Through this approach, we obtain further insights into the structure of the optimal event-triggering law. It should be noted that the optimal control policy $\gamma^{*}$ is fully determined, when fixing parameters $\alpha_{\tau_{k}, k}$. This implies that the side-channel transmitting $u_{k}$ to the event-trigger is not needed anymore, as it can be computed directly at the event-trigger with its full measurement knowledge. Furthermore, we obtain the following lemma.

Lemma 5: Let $\alpha_{\tau_{k}, k}$ be fixed for all $k, \tau_{k} \in\{0, \ldots, N-1\}$. Then, the estimation discrepancy defined as

$$
e_{k}=\hat{x}_{k \mid k}^{\mathcal{E}}-\mathrm{E}\left[x_{k} \mid \mathcal{I}_{k}^{\mathcal{C}}, \delta_{k}=0\right]
$$

and $\tau_{k}$ are a sufficient statistics for the optimal eventtriggering law $\mathrm{f}_{k}$ for all $k \in\{0, \ldots, N-1\}$.

Proof: For notational convenience, we define

$$
\left\|x_{k}-\hat{x}_{k \mid k}^{\mathcal{C}}\right\|_{\Gamma_{k}}^{2}=\left(x_{k}-\hat{x}_{k \mid k}^{\mathcal{C}}\right)^{\mathrm{T}} \Gamma_{k}\left(x_{k}-\hat{x}_{k \mid k}^{\mathcal{C}}\right) .
$$

Assume $\hat{x}_{k \mid k}^{\mathcal{C}}$ be given by Lemma 4 with fixed $\alpha_{\tau_{k}, k}$ for all $k$ and $\tau_{k}$. By considering $\mathcal{I}_{k}^{\mathcal{E}}$ to be the information state with

$$
\mathcal{I}_{k+1}^{\mathcal{E}}=\left(\mathcal{I}_{k}^{\mathcal{E}}, y_{k+1}, u_{k}, \delta_{k}\right)
$$

we can apply the dynamic programming algorithm [15]

$$
\begin{aligned}
J_{k}\left(\mathcal{I}_{k}^{\mathcal{E}}\right)=\min _{\delta_{k} \in\{0,1\}} \mathrm{E}[ & \left\|x_{k}-\hat{x}_{k \mid k}^{\mathcal{C}}\right\|_{\Gamma_{k}}^{2}+\lambda \delta_{k}+ \\
& \left.+J_{k+1}\left(\mathcal{I}_{k}^{\mathcal{E}}, y_{k+1}, u_{k}, \delta_{k}\right) \mid \mathcal{I}_{k}^{\mathcal{E}}, \delta_{k}\right]
\end{aligned}
$$


with $J_{N}\left(\mathcal{I}_{N}^{\mathcal{E}}\right)=0$ to solve optimization problem (13).

The running costs for $\delta_{k}=1$ can be written as

$$
\mathrm{E}\left[\left\|x_{k}-\hat{x}_{k \mid k}^{\mathcal{E}}\right\|_{\Gamma_{k}}^{2} \mid \mathcal{I}_{k}^{\mathcal{E}}, \delta_{k}=0\right]+\lambda .
$$

For $\delta_{k}=0$ the running costs are

$\mathrm{E}\left[\left\|x_{k}-\hat{x}_{k \mid k}^{\mathcal{E}}\right\|_{\Gamma_{k}}^{2} \mid \mathcal{I}_{k}^{\mathcal{E}}, \delta_{k}=1\right]+\left\|\hat{x}_{k \mid k}^{\mathcal{E}}-\mathrm{E}\left[x_{k} \mid \mathcal{I}_{k}^{\mathcal{C}}, \delta_{k}=0\right]\right\|_{\Gamma_{k}}^{2}$

The term $\mathrm{E}\left[\left\|x_{k}-x_{k \mid k}^{\mathcal{E}}\right\|_{\Gamma_{k}}^{2} \mid \mathcal{I}_{k}^{\mathcal{E}}, \delta_{k}\right]$ in the running costs is independent of $\delta_{k}$ because of Lemma 3. Therefore, for $k=$ $N-1$, the optimal $\mathrm{f}_{N-1}$ is determined by

$$
J\left(\mathcal{I}_{N-1}^{\mathcal{E}}\right)=\min _{\delta_{N-1}}\left(\left\|e_{N-1}\right\|_{\Gamma_{N-1}}^{2}, \lambda\right)
$$

where $e_{N-1}=\hat{x}_{N-1 \mid N-1}^{\mathcal{E}}-\mathrm{E}\left[x_{N-1} \mid \mathcal{I}_{N-1}^{\mathcal{C}}, \delta_{N-1}=0\right]$. We observe that the value function $J_{N-1}$ may be expressed as a function of $e_{N-1}$. Subsequently, the evolution of the error signal $e_{k}$ is derived. For $\delta_{k}=1$, we have

$$
\begin{aligned}
& e_{k+1}=\hat{x}_{k+1 \mid k+1}^{\mathcal{E}}-\mathrm{E}\left[x_{k+1} \mid \mathcal{I}_{k+1}^{\mathcal{C}}, \delta_{k+1}=0\right] \\
& =A \hat{x}_{k \mid k}^{\mathcal{E}}+B u_{k}+\Sigma_{k+1 \mid k+1}^{\mathcal{E}} C^{\mathrm{T}}\left(y_{k+1}-C A \hat{x}_{k \mid k}^{\mathcal{E}}-C B u_{k}\right) \\
& \quad-A \hat{x}_{k \mid k}^{\mathcal{E}}-B u_{k}-\alpha_{\tau_{k}, k}
\end{aligned}
$$

For $\delta_{k}=0$, we have

$$
\begin{aligned}
& e_{k+1}=\hat{x}_{k+1 \mid k+1}^{\mathcal{E}}-\mathrm{E}\left[x_{k+1} \mid \mathcal{I}_{k+1}^{\mathcal{C}}, \delta_{k+1}=0\right] \\
& =A \hat{x}_{k \mid k}^{\mathcal{E}}+B u_{k}+\Sigma_{k+1 \mid k+1}^{\mathcal{E}} C^{\mathrm{T}}\left(y_{k+1}-C A \hat{x}_{k \mid k}^{\mathcal{E}}-C B u_{k}\right) \\
& \quad-A \hat{x}_{k \mid k}^{\mathcal{C}}-B u_{k}-\alpha_{\tau_{k}, k}
\end{aligned}
$$

Summarizing Equations (18) and (19) yields

$$
\begin{aligned}
e_{k+1}= & \left(1-\delta_{k}\right) A e_{k}-\alpha_{\tau_{k}, k} \\
& +\Sigma_{k \mid k}^{\mathcal{E}} C^{\mathrm{T}}\left(y_{k+1}-C A \hat{x}_{k \mid k}^{\mathcal{E}}-C B u_{k}\right)
\end{aligned}
$$

The state estimate $\hat{x}_{k \mid k}^{\mathcal{E}}$ is obtained by (11) and the evolution of $\tau_{k}$ is given by

$$
\tau_{k+1}= \begin{cases}\tau_{k} & \delta_{k}=0 \\ k & \delta_{k}=1\end{cases}
$$

In the following, we prove that $\left(e_{k}, \tau_{k}\right)$ is sufficient to derive the statistical properties of the value function, by using induction. We have already seen in Equation (17) that this is true for $k=N-1$. Assuming that $J_{k+1}$ is a function of $\left(e_{k+1}, \tau_{k+1}\right)$, we show that $J_{k}$ is a function of $\left(e_{k}, \tau_{k}\right)$. Due to Equations (15) and (16) the running costs are functions of $e_{k}$. It remains to calculate the conditional expectation of $J_{k+1}$ given $\left\{\mathcal{I}_{k}^{\mathcal{E}}, \delta_{k}\right\}$ The variables, $\tau_{k+1}$ and $u_{k}$ are measurable with respect to $\left\{\mathcal{I}_{k}^{\mathcal{E}}, \delta_{k}\right\}$. The remaining issue is the calculation of the probability distribution of $e_{k+1}$ given $\left\{\mathcal{I}_{k}^{\mathcal{E}}, \delta_{k}\right\}$. By Equation (20), we observe that $e_{k+1}$ is Gaussian given $\left\{\mathcal{I}_{k}^{\mathcal{E}}, \delta_{k}\right\}$ with predetermined covariance $\Sigma_{k \mid k}^{\mathcal{E}}$, which is independent of $\delta_{k}$, and mean determined by $e_{k}, \tau_{k}$ and $\delta_{k}$. Therefore, we conclude, $J_{k}$ may be expressed by $e_{k}, \tau_{k}$ and $\delta_{k}$, which completes the induction and proves above lemma.
Remark 7: Summarizing Lemma 1-5, we can state: The controller consists of an affine-linear state predictor and linear gains $L_{k}$. The event-trigger consists of a Kalman filter and a copy of the affine-linear predictor at the controller. The resulting error discrepancy $e_{k}$ and the time step of the last transmission $\tau_{k}$ determine, whether the event-trigger transmits the actual Kalman estimate to the controller.

Remark 8: If the bias $\alpha_{\tau_{k}, k}$ are assumed to be zero, the obtained results in this paper are in accordance with [14]. In [14], it is assumed that the event-trigger is a threshold policy, which is a function of $e_{k}$ that is point-symmetric to the origin. It can be seen easily from Lemma 5 that by choosing $\alpha_{\tau_{k}, k}=0$ for all $\tau_{k}$ and $k$, the resulting policy will be a threshold policy, where the threshold is point symmetric to the origin for every $k$. On the other hand, Lemma 1-5 support the assumptions made in [14] by giving it an additional interpretation on optimality through cost function (3). This also applies to the control systems developed in [6], [7], [13], where controllers are presumed to be linear controllers with state estimators, and the event-triggers are also threshold policies on the estimation discrepancy between controller and at event-trigger.

\section{NUMERICAL ILLUSTRATION}

The obtained characterization of the optimal eventtriggered controller enables the construction of an algorithm that obtains the optimal solution. The algorithm can be summarized as follows:

1) Calculate the control gains $L_{k}$ and matrices $P_{k}, \Gamma_{k}$, $\Sigma_{k \mid k}^{\mathcal{E}}$ given in Lemma 1, by Equation (14) and in Remark 4.

2) Determine the optimal cost $J$ for every fixed parameters $\alpha_{\tau_{k}, k}$ by using dynamic programming.

3) The parameters $\alpha_{\tau_{k}, k}$ with minimal cost $J$ yield the optimal event-triggered controller.

The search for finding the optimal parameter set $\alpha_{\tau_{k}, k}$ is numerically tedious as the number of parameter grows quadratically with the time horizon $N$. It should be noted, however, that the optimal event-triggering law and control law can be computed offline. During execution time the event-trigger computes the state estimate via Kalman filtering and decides upon the vector $\left(e_{k}, \tau_{k}\right)$, whether to transmit the state estimate to the controller. Thus, online computations within the event-trigger are numerically feasible, which makes it attractive for systems with limited computational power at sensor nodes.

For illustrative purpose, we give a numerical example of a scalar system and display the optimal eventtriggering law. Consider the system given by Equation (1) with $A=1, B=1$ and $C=1$. The covariance $S$ of the initial state $x_{0}$ is 1 and its mean can be chosen arbitrary as the cost in (13) is independent of $x_{0}^{\mathrm{m}}$. The cost function has parameters $Q_{N}=Q=1, R=10$ and $\lambda=10$ with horizon $N=5$. An exhaustive search over the parameters $\alpha_{\tau_{k}, k}$ showed for this configuration that the minimal costs are 
achieved, when $\alpha_{\tau_{k}, k}=0$ for all $\tau_{k}$ and $k$. As already stated in Remark 7 , the resulting event-triggering policy is symmetric and in addition independent of $\tau_{k}$, as all $\alpha_{\tau_{k}, k}$ are equal. The optimal event-triggering law is calculated numerically by uniform spatial gridding of $e_{k}$ and is drawn in Figure 3. The value function for time step $k=0$ is illustrated in Figure 4. It remains an open problem to show, whether the optimal parameters $\alpha_{\tau_{k}, k}$ are zero for any arbitrary configuration.

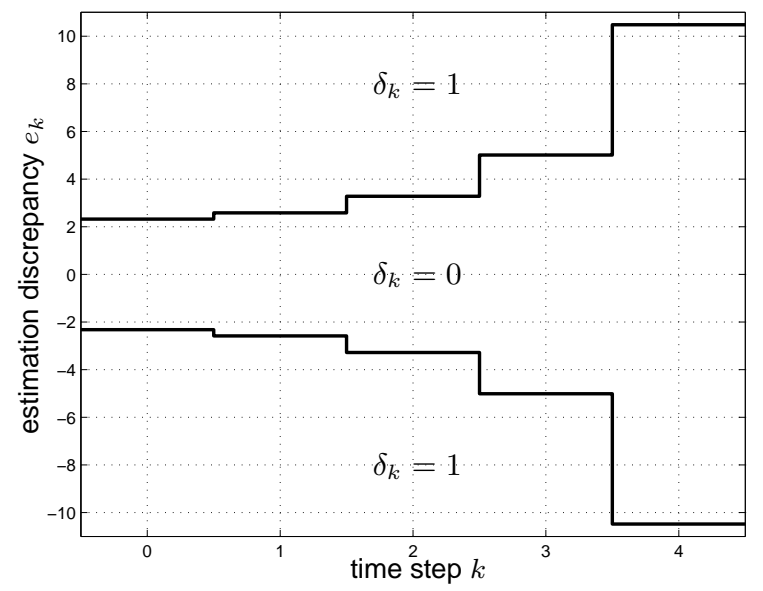

Fig. 3. Optimal event-trigger policy $\mathrm{f}^{*}$ with indicated thresholds.

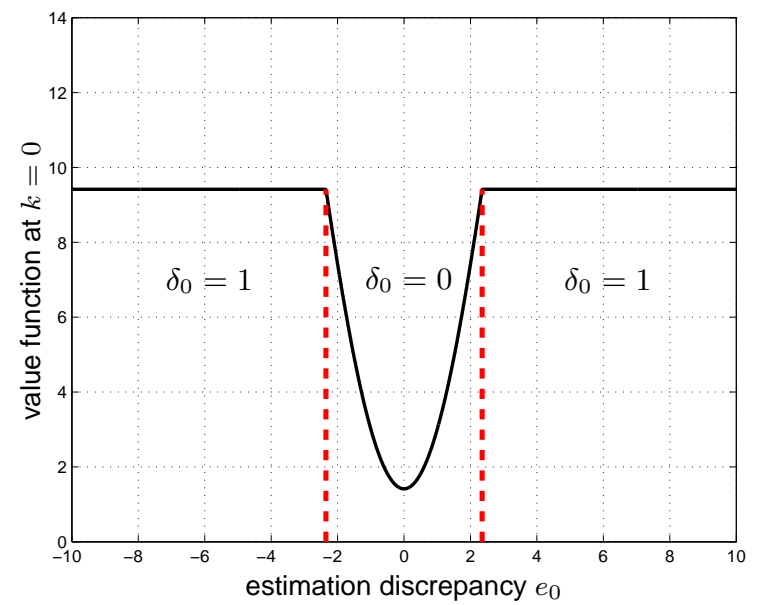

Fig. 4. Value function at time $k=0$ with indicated thresholds.

\section{CONCLUSIONS}

This paper has addressed the problem of designing optimal event-triggered controllers under costly observations. By reformulating the proposed optimization problem, it is possible to characterize optimal event-triggered controllers for linear systems penalizing the transmission of sensor information. The optimal controller is affine linear with an additional bias term in the estimator. The optimal event-trigger depends on the difference between the state estimates at controller and event-trigger. This characterization enables the systematic design of optimal event-triggered controllers. In addition, it provides interpretations with respect to optimality for existing results that propose the same type of controller. Further investigations concern the properties of the bias terms in the state estimator at the controller which will allow an efficient calculation of the optimal solution. Future research aims at the application of the obtained results to multi-loop control systems embedded in contention-based networks.

\section{ACKNOWLEDGMENTS}

This work was supported in part by the German Research Foundation (DFG) within the Priority Program SPP 1305 "Control Theory of Digitally Networked Dynamical Systems".

\section{REFERENCES}

[1] K. Åström and B. Bernhardsson, "Comparison of Riemann and Lebesgue sampling for first order stochastic systems," Decision and Control, 2002, Proceedings of the 41st IEEE Conference on, vol. 2, pp. 2011-2016, Dec. 2002.

[2] A. Cervin and T. Henningsson, "Scheduling of event-triggered controllers on a shared network," in Proc. 47th IEEE Conference on Decision and Control, (Cancun, Mexico), 2008.

[3] J. S. Baras, P. Hovareshti, and S. Perumal, "Event Triggered Distributed Collaborative Control," in Proc. European Control Conf. (ECC), (Budapest, Hungary), Aug. 2009.

[4] D. V. Dimarogonas and K. H. Johansson, "Event-triggered Cooperative Control," in Proc. European Control Conf. (ECC), (Budapest, Hungary), Aug. 2009.

[5] P. Wan and M. D. Lemmon, "Distributed Network Utility Maximization using Event-triggered Barrier Methods," in Proc. European Control Conf. (ECC), (Budapest, Hungary), Aug. 2009.

[6] Y. Xu and J. Hespanha, "Optimal communication logics in networked control systems," Decision and Control, 2004. CDC. 43rd IEEE Conference on, vol. 4, pp. 3527-3532 Vol.4, Dec. 2004.

[7] Y. Xu and J. P. Hespanha, "Communication logic design and analysis for networked control systems," in Current trends in nonlinear systems and control (L. Menini, L. Zaccarian, and C. T. Abdallah, eds.), Boston: Birkhäuser, 2006

[8] M. Rabi and J. S. Baras, "Level-triggered control of a scalar linear system," Control and Automation, 2007. MED 07. Mediterranean Conference on, pp. 1-6, 27-29 June 2007.

[9] M. Rabi, K. H. Johansson, and M. Johansson, "Optimal stopping for event-triggered sensing and actuation," Decision and Control, 2008 47th IEEE Conference on, Dec. 2008.

[10] O. C. Imer and T. Basar, "Optimal estimation with limited measurements," Decision and Control, 2005 44th IEEE Conference on, vol. 2, pp. 1029-1034, 2005.

[11] O. Imer and T. C. Basar, "Optimal control with limited controls," American Control Conference, 2006. Proceedings of the 2006, pp. 298-303, June 2006.

[12] A. Molin and S. Hirche, "On LQG joint optimal scheduling and control under communication constraints," in Proc. 48th IEEE Conference on Decision and Control, (Shanghai, China), 2009.

[13] J. K. Yook, D. M. Tilbury, and N. R. Soparkar, "Trading Computation for Bandwidth: Reducing Communication in Distributed Control Systems Using State Estimators," IEEE Trans. on Control Systems Technology, vol. 10, pp. 503-518, July 2002.

[14] C. Ramesh, H. Sandberg, and K. H. Johansson, "LQG and medium access control," in 1st IFAC Workshop on Estimation and Control of Networked Systems, 2009.

[15] D. P. Bertsekas, Dynamic programming and optimal control. Vol. I. 3rd ed., Athena Scientific, Belmont, MA, 2005.

[16] K. J. Åström, Introduction to Stochastic Control Theory. Dover Publications, 2006 